\title{
Public perceptions of fruit and vegetable consumption: qualitative analysis of online comments
}

\author{
B. Hornsby and H. Ensaff \\ School of Food Science and Nutrition, University of Leeds, Leeds, LS2 9JT
}

Only $27 \%$ of adults aged $19-64$ years and $35 \%$ of older adults $65+$ years meet the UK '5 A Day' (400g) guidelines for fruit and vegetable (FV) consumption ${ }^{(1)}$. Previous research has identified multiple factors influencing FV consumption, with time, sensory attributes and variety acting as potential barriers ${ }^{(2)}$. The aim of this qualitative study was to explore perceptions and perspectives on the consumption of fruit and vegetables, using online users' comments. Analysis of online comments in response to media articles has been used previously in the exploration of public opinions on public health issues ${ }^{(3)}$.

This study's data comprised online comments related to one specific study ${ }^{(4)}$, a meta-analysis examining FV intake, which received high media coverage and online comments in response. The online comments originated from the top $10 \mathrm{UK}$ online media publications according to pageviews. Overall, 2696 comments (103,930 words) were collected and imported into NVivo 11 software (QSR International), which was used for the data exploration and analysis. An inductive thematic approach ${ }^{(5)}$ was adopted for the analysis and coding of the data was reviewed in an iterative process.

Four overarching themes emerged from the initial analysis:

1. Food Landscape and how this influenced FV consumption was important for many people; often this included cost: "...veg is expensive, the working poor can't afford ten of them a day" and the quality and variety of FV available: “...limited choice, very poor quality."

2. Personal Factors emerged as a main theme and many comments related to perceived barriers to FV consumption. These included parents having to consider their children: "It's hard to get children to each much fruit and veg". Taste preferences and the time, effort and skills needed were also mentioned: "Would not have enough hours in the day to fit this lot in!"

3. Rejection, interestingly, was identified as a main theme and often it was in the form of ridicule, typified by comments related to alcohol: “. . does cider count for my apple intake?", fast food and enjoyment. Likewise, scepticism surrounding scientific research was evident: ". . . no doubt the news tomorrow will be that eating 10 a day gives you cancer". A quality versus quantity of life debate also formed the basis of rejection in some of the discussions.

4. Lack of Knowledge was evident from users' comments and this included confusion surrounding the current FV guidelines, e.g. which foods count and what a portion size is: "They really, really, really need to stop using the word 'portions' and 'servings'. They are vague and mean little to most punters." Likewise there was evidence of limited knowledge on diet and health outcomes, and a misunderstanding of the scientific research.

This analysis revealed gaps in the understanding of the FV guidelines and suggest that nutritional education surrounding FV portion sizes and the importance of FV variety should be forthcoming. Additionally, targeted health interventions addressing individual barriers such as food skills should also be considered.

1. Bates B, Cox L, Nicholson S, et al. (2016) National Diet and Nutrition Survey Results from Years 5 and 6 (combined) of the Rolling Programme (2012/2013 - 2013/2014). London: Public Health England.

2. Krølner R, Rasmussen M, Brug J, et al. (2011) Determinants of fruit and vegetable consumption among children and adolescents: a review of the literature. Part II: qualitative studies. Int. J. Behav. Nutr. Phys. Act. 8, 112.

3. Giles EL, Holmes M, McColl E, et al. (2015) Acceptability of financial incentives for breastfeeding: thematic analysis of readers' comments to UK online news reports. BMC Pregnancy Childbirth 15, 116. BioMed Central.

4. Aune D, Giovannucci E, Boffetta P, et al. (2017) Fruit and vegetable intake and the risk of cardiovascular disease, total cancer and all-cause mortality - a systematic review and dose-response meta-analysis of prospective studies. Int. J. Epidemiol. 46, 1029-1056. Oxford University Press.

5. Thomas DR (2006) A General Inductive Approach for Analyzing Qualitative Evaluation Data. Am. J. Eval. 27, $237-246$. 